\title{
Active SAR Antennas: Design, Development, and Current Programs
}

\author{
P. Capece \\ Thales Alenia Space Italia, Via Saccomuro 24, 00131 Roma, Italy \\ Correspondence should be addressed to P. Capece, pasquale.capece@thalesaleniaspace.com \\ Received 17 October 2008; Revised 2 January 2009; Accepted 11 February 2009 \\ Recommended by Giovanni Toso
}

\begin{abstract}
The paper presents the design guidelines for Active SAR Antennas followed for the development of COSMO Sky Med SAR Antenna Subsystem. They have brought to define the antenna architecture, the RF, and electrical requirements for all the antenna RF, digital and power units, taking into account the available technologies and achievable performance. The antenna design is described for what the main subsystems is concerned, such as RF, power, digital, including mechanical and thermal aspects. Finally the paper reports also a brief description of the most recent development carried out in Canada and in Europe in the last ten years, including the on-going programs.
\end{abstract}

Copyright () 2009 P. Capece. This is an open access article distributed under the Creative Commons Attribution License, which permits unrestricted use, distribution, and reproduction in any medium, provided the original work is properly cited.

\section{SAR Antenna Requirements and Design}

The SAR Antenna requirements include several RF, electrical, mechanical, and thermal parameters. The most important are the required beam shaping and steering capabilities, which implies the necessity to consider an active-phased array instead of alternative solutions such as multifeed reflector antennas, or passive-phased array, which do not have the required flexibility. Available technology for radiating linear array, Transmit/Receive (T/R) modules, and electronics strongly impacts other fundamental parameters such as the mass, the RF efficiency, the DC power efficiency, and the thermal dissipation. The consequence is that the antenna dimensioning will be the best compromise among all the mentioned aspects.

Main RF requirements to be considered are the following:

(i) the beam size in azimuth and the minimum elevation beamwidth,

(ii) the beam shaping capability,

(iii) the azimuth and elevation steering angles,

(iv) the central frequency and the bandwidth,

(v) the EIRP,

(vi) the noise figure,

(vii) dual polarization.
As consequence the following aspects have to be fixed:

(i) the technology of the radiating elements, that has some impacts also the mechanical and thermal designs of the whole system,

(ii) RF subsystem, including passive and active units,

(iii) digital subsystem,

(iv) power subsystem,

(v) mechanical design,

(vi) thermal design.

Moreover it is necessary to divide the antenna in electronics subassemblies to allow the production chain to guarantee the needed rate and to perform all the required acceptance test in short time.

1.1. RF Technologies. Central frequency, bandwidth, ohmic losses, cross-polarization purity, but also mass and manufacturing cost, impacts on the selection of the technology used to realize the linear array. Typically two technologies have been used up to now for the RF radiator of SAR Antennas: slotted waveguides and linear array of patch elements. The first presents very good performance in terms of ohmic losses and cross-purity, but it is applicable only for bandwidth up to $2 \%$, and have as major drawback a strong mutual coupling phenomena, which implies accurate modelling of 
the antenna surface to control the antenna sidelobes and to avoid high active mismatch. The electromagnetic design becomes complex in case dual polarization is needed. In this case different waveguides design has to be considered for the vertical and horizontal polarizations and the two waveguides, needed to realize the dual polarization, have to fit in the elevation steering spacing which is typically in the order of $0.7 / 075$ wavelength. Finally the mass can be considered acceptable in case of carbon fiber waveguides are considered and for frequencies of $5 / 10 \mathrm{GHz}$ or higher. Linear array of patches is simpler to be designed and manufactured, cheap and lightweight, presents larger bandwidths (up to $4 / 5 \%$ or more for accurate design). Cross-level can be improved by sequential rotation techniques, while the major drawback consists in higher ohmic losses due to the microstrips used for the RF Beamformers. Patch array can be used from lower frequency ( $\mathrm{P}$ band) up to $\mathrm{X}$ band. At higher frequency only an accurate design and manufacturing can improve the cross-polarization purity and maximize the efficiency, while eliminating the glues from microstrips surface it is possible to reduce the ohmic losses of the whole assembly.

Concerning the EIRP and the noise figure, they strongly depend on the amplifiers performance. For such antennas distributed $\mathrm{T} / \mathrm{R}$ modules are used to maximize the antenna aperture control to shape and steer the antenna beam. The TR module typically consists of a hybrid which includes a Tx channel and an Rx channel, each having few chips per channel. Multipack hybrids with 2/4 or $8 \mathrm{Tx}$ and $\mathrm{Rx}$ channels have been also studied and produced in the past, but the experience demonstrated that these configurations, have a worst yeld and cannot reduce the antenna cost.

Packaging and substrates are an important issue for mass production; thick film and thin film technologies over alumina/multilayer ceramics have been largely used in the past. A single LTCC substrate is today the baseline for TR module packaging, reducing the number of steps and the cost in production. Another important aspect is the electrical connections to the external circuits and the RF I/Fs. RF Blind mate connectors and solder-less contacts are adopted to allow a simple mounting/dismounting of the module also during the assembly and testing phases.

The Tx section of the module is typically based on a cascade of two chips in GaAs technology: the RF driver and the HPA. Chips based on HBT and P-HEMPT technologies are the most used for the final power chip. The available output power depends on the frequency and the chip dimension. It can be used as single chip or in tandem configuration to reach the wanted power. Typical Pout of an HPA depends on the central frequency: 10/12 W are today available for $\mathrm{C}$ band, which reduces to $8 / 10 \mathrm{~W}$ for the $\mathrm{X}$ band. Concerning the receive path, the noise figure is affected by the limiter needed to protect the LNA from unwanted reflected high power caused by open/short circuits. Achievable Figures are in the range of $3 / 3.5 \mathrm{~dB}$ for the mentioned frequencies.

The amplitude and phase control can be obtained by used separated attenuator/phase shifter chips or a multifunctional chip (core chip), which includes both the controls plus amplification and switching capabilities. These controls are used both to select the amplitude and phase of the module, in Tx and in Rx mode, but also to compensate the amplitude and phase changes caused by the temperature variation on the whole RF chain in the applicable thermal range. In fact in space, for large active antennas, it is very difficult to stabilize the operative temperature, especially when high dissipation is generated internally by the antenna electronics. As consequence it is necessary to compensate the gain and phase variation with temperature of the TR modules by an external digital controller which receive the wanted setting from a central beam controller and, on the basis of the temperature of the module, sets the amplitude and phase steps to stabilize the overall amplitude gain and phase.

1.2. Electronic Front End. In order to allow the TR modules to work correctly, two additional conditioning circuits are needed:

(1) a digital I/F able to receive/transmit the data from the Tile controller and to implement electronic protection of the hybrids; this is achieved by a dedicated ASIC, which reduces the circuit complexity;

(2) a power section, which includes the capacitor bank to work in pulsed way, and the high-current switches, to switch on/off the RF Tx and Rx chains.

These circuits can be placed inside each the TR module, or can be mounted on dedicated PCB externally to drive a group (4 or 8) TR modules. The whole assembly is named (Electronic Front End) EFE and includes also the RF combiners/dividers for the TR modules. The EFE hybrids directly interface the antenna radiators and the RF dividers through the blind mate connectors placed on the bottom of the module, while digital controller and power supply are interfaced on the other two narrow sides of the hybrid module.

1.3. True Time Delay Lines and Array Bandwidth. Also if all the SAR antennas are typically based on RF distribution networks equally phased in the whole frequency band by equal RF paths from the antenna input port to the TR modules, the frequency bandwidth of a large-phased array is limited by the beam pointing variation when $0-360^{\circ}$ degrees phase shifters are used to generate the aperture phase slope to steer the beam. As reported in [1], the beam pointing changes with the frequency for a given phase setting to the TR modules phase shifters: the pointing angle at frequency $f$ is given by the simple formula

$$
\sin (\theta)=\left(\frac{f_{0}}{f}\right) * \sin \left(\theta_{0}\right),
$$

being: $\theta$ the pointing at the frequency $f ; f_{0}$ the central frequency; $\theta_{0}$ the pointing at $f_{0}$.

In Figure 1 the beam pointing versus frequency variation at different steering angles is reported for a generic-phased array. For SAR Antennas with a typical bandwidth of $2 \%$ and $15^{\circ}$ of steering angle along the elevation plane, the pointing variation expected will be in the order of $0.3^{\circ}$. This value has to be compared with the antenna beamwidth along 


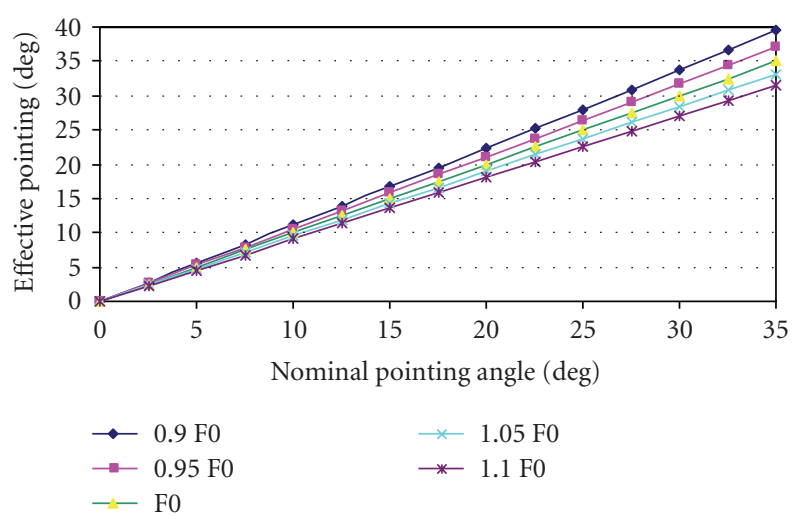

FIGURE 1: Beam pointing versus frequency in degrees.

that plane. The pointing variation is due to the fact that a constant phase distribution is applied by the phase shifters in the whole bandwidth, while the nominal phase distribution should change with the frequency to maintain the same beam pointing.

If the pointing variation is small with respect to the beamwidth, in the order of $5-10 \%$, it can be tolerated, while if the beam pointing variation corresponds to an important fraction of the beamwidth, it is necessary to introduce True Time Delay Lines (TTDL) in the beam forming network, each acting over a part of the antenna surface. The TTDL realizes a variable delay, typically in terms of multiple of wavelength, according to the steering angle and the position of the subarrays, implementing a phase slope versus frequency which allow to correct the phase distribution over the antenna surface in the frequency band. Also in case true time delay lines are used, the residual beam pointing variation with the frequency is an inverse function of the size of the subarray fed by a single true time delay line and of the minimum step of the TTDL. Fast switching is necessary in case fast beam change or steering is required. As switches, ferrite circulators can be used with waveguide technology, or more integrated solution based on MMICs switches and microstrip lines can be also adopted, but they require additional amplification.

1.4. Digital Subsystem. The objective of the digital subsystem is to control the setting of the TR modules in order to allow the antenna to generate the wanted beam. It consists of a central beam controller which knows all the wanted settings for each TR module inside the antenna for each desired beam, and a number of distributed controllers which directly act on a group of TR modules (typically from 16 to 32) to set the modules, implementing also the thermal compensation.

The central antenna controller communicates to the distributed secondary controllers all the wanted setting; the secondary controllers send to the TR modules the setting data suitable corrected to take into account the TR module temperature. It is important to note that the compensation loop acts almost in real time in order to minimize the $\mathrm{RF}$ drift of the device in the thermal range. Typically the control loop for temperature compensation is performed in fractions of a second, and it is based on a look-up table which contains the compensation data for each TR module. In case of TTDLs are present in the antenna BFN, they also need to be compensated versus temperature, and this is achieved still acting on the TR module level setting (the TR modules amplitude and phase are used to compensate also the TTDL variation versus temperature).

The digital controller is internally or externally redounded, considering that all the $\mathrm{TR}$ modules driven by the controller will be lost in case of the controller failure.

1.5. Power Subsystem. The power subsystem is based on a suitable dimensioning of the satellite power system, such as the battery and the solar arrays, a dedicated unit which main function is to switch on/off and filtering the several distributed power supplies of the antenna panels and, finally, by the capacitor banks placed on the EFE cards. The continuous switch on/off of the TR modules with the pulse repetition frequency generates a ripple on the power bus that can disturb other units. As consequence the control loop of the power supply has to be adjusted in order to avoid to much strong current ripple on the primary bus, but also to limit as possible the voltage variations on the TR modules that can affect the HPA performance. Filtering circuit is close to the switching section in order to reduce the emission towards the battery. The number of power supplies on the antenna depends on the number of TR modules to be fed, their power consumption, the duty cycle, the reliability performance, and the total number of TR modules in the antenna. In case of a large antenna is considered, the number of power supplies can reach several tens. If the reliability Figure of the single power supply is able to guarantee that only one or two units over the whole number could be lost in the operative life, it is possible to avoid the redundancy of the power supplies, saving at the same time cost and mass.

1.6. Mechanical Design. The SAR antenna has a large aperture of several square meters and typically is divided into mechanical panels that are in stowed condition at the launch and then deployed when the satellite has reached the final orbit. This implies the presence of hold down and release mechanisms, able to take in stowed configuration the antenna during the launch and to support the mechanical loads in this phase, and deployment mechanisms to open the antenna when it is in flight. Concerning the panels, two configurations are possible: (1) the electronics are integrated in subassemblies (the Tiles), which are mounted on a panel supporting frame; (2) all the electronic is integrated in large and closed panel structures.

An alternative configuration is based on Tiles directly mounted on a sidewall of the satellite: this implies a spacecraft having a length close to the antenna dimension, that can occur only for antenna not longer than $4 / 5 \mathrm{~m}$.

Antennas having a length of $5 / 7 \mathrm{~m}$, can be organized in two or three mechanical panels, with the two external ones are deployable.

In case of longer antennas of a dimension of 10/12 m, the number of mechanical panels increases up to 4 or 5 . In 


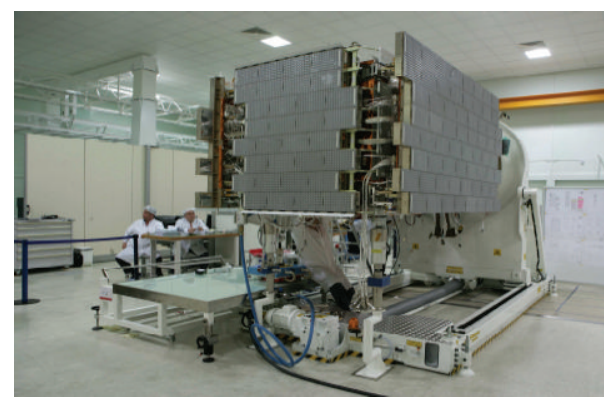

(a)

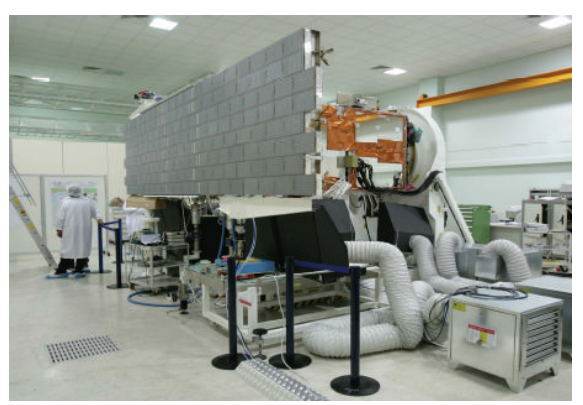

(b)

Figure 2: COSMO sky med active SAR antenna (stowed \& deployed).

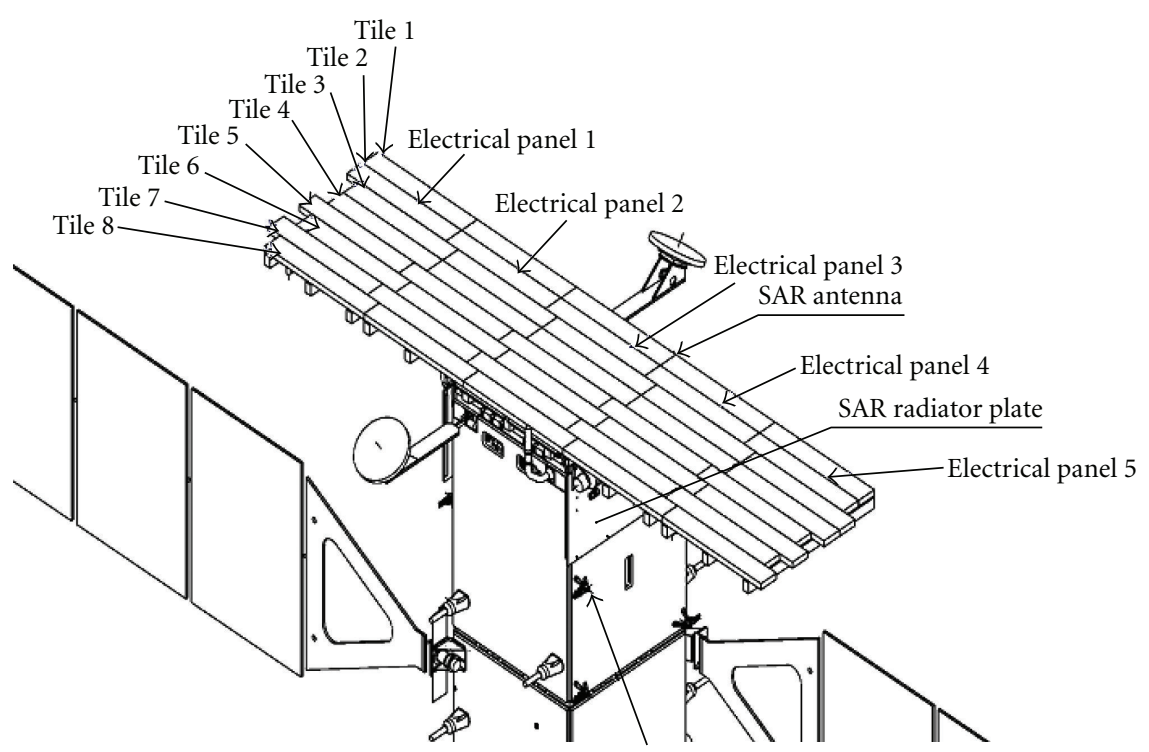

Figure 3: Antenna organization in tiles.

this case couples of panels can be grouped to realize lateral wings, each having a double deployment. The hold down and release mechanisms are typically based on pyrocutter devices, while the deployment mechanisms can be driven by motors or springs. In case of motors are used, driver electronic unit is also necessary to control the deployment, while spring-based mechanisms are mechanically controlled by dumpers and do not need control electronics.

One of the most critical aspects for mechanical design is to guarantee the antenna planarity and therefore reduced thermal distortion during the antenna functioning, to guarantee patterns stable performance. The amount of acceptable distortion of the antenna plane is inverse to the RF frequency of the antenna; typically $1.5 / 2 \mathrm{~mm}$ is still acceptable for $\mathrm{X}$ band, while $3 / 4 \mathrm{~mm}$ can be tolerated in $\mathrm{C}$ band.

The use of very low (Coefficient of Thermal Expansion) CTE material for Tiles and supporting frames, such as carbon fiber reinforced plastic (CFRP), having a CTE in the order of 1-2 parts per million (ppm) per ${ }^{\circ} \mathrm{K}$, improves the dimensional stability at the expense of poorer thermal conductivity due to low thermal conductivity of the resins.
Aluminium, with good thermal conductivity, is also largely used for these antennas, improving, with respect to the CFRP, the heat transfer from the TR modules to the main frames and towards to the thermal radiators areas. Nevertheless higher CTE (24-25 ppm per $\left.{ }^{\circ} \mathrm{K}\right)$ implies that, in case of different temperature between the upper surface and bottom side of the antennas, the aperture surface can be warped along the azimuth plane (thermal distortion). This problem can be solved by accurate thermal design of the heat vias from the hot to the cold areas of the antenna. Heat pipes can be also used to minimize all the thermal gradients and the thermal distortion.

1.7. Thermal Design. The thermal design is of particular importance because it has to guarantee the limited thermal excursion as applicable to electronic devices inside the antenna, considering the required continuous functionality.

The thermal configuration study will optimize the working thermal range of the antenna and the heater power system demand, considering the functional requirements such as and the number of images per orbit, duration of 


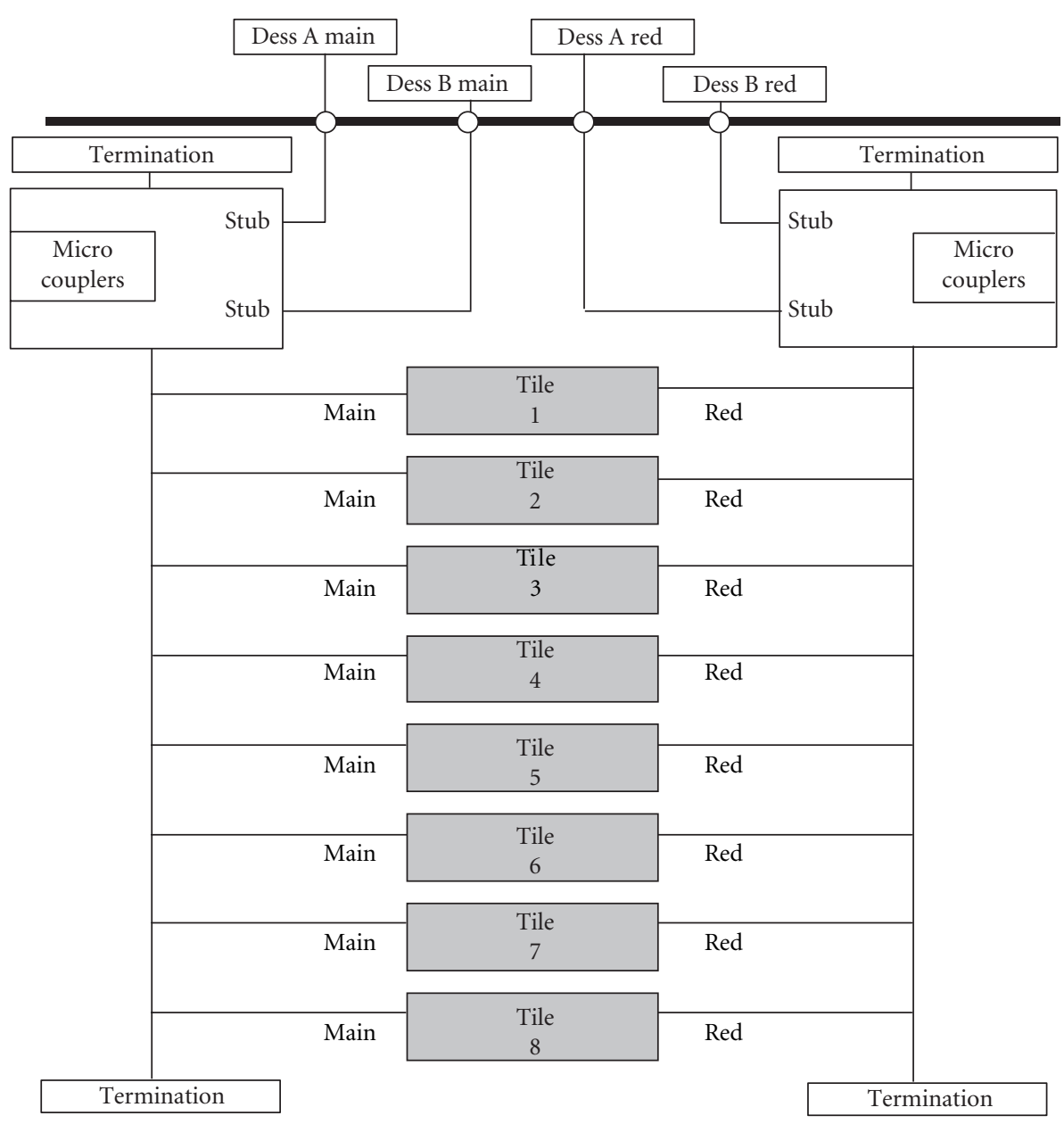

FIgURE 4: 1553 digital busses for each column of 8 tiles.

the continuous acquisition in image modes, and single or dual looking operations which can determine a rapid grow up of the temperature. The fast temperature increment is controlled mainly from the thermal capacity of the antenna, in particular by the parts which are in good thermal contact with the hot spots of the electronics (the TR modules and power supplies). Then the accumulated heat can be dissipated by the front and/or back side during the rest of the orbit.

An important aspect of the thermal design is the capability of the antenna to work in front to sun or back to sun condition. In the first case the heat dissipation can occur mainly through the back side of the antenna, while the heat from the sun can be limited by using RF transparent sunshield. The major drawback for this configuration is that the satellite body blocks the dissipation of the central panels or parts of the lateral panels, as consequence heat pipes and dedicated thermal radiators have to be used to remove the heat.

When exposed to the sun with the back side, the antenna can radiate through the radiating linear arrays, if their thermal conductivity and surface is adequate. The best case occurs when the antenna is able to work in both the two cases, improving the revisit time of the mission.

\section{In Flight Active Phased Array SAR Antennas}

The above design considerations are fully applicable to the SAR antennas developed in the last years for Earth Observation missions.

Hereafter the description of the antenna designed, manufactured, and tested by Thales Alenia Space Italy for the COSMO Sky Med mission is reported. Moreover, other similar antennas developed in Europe and Canada, in the last ten years, are also briefly described and discussed.

\subsection{COSMO Sky Med Antenna. COSMO-SkyMed (Constel-} lation of Small Satellites for Mediterranean basin Observation) is a 4 -satellite constellation funded by (Agenzia Spaziale Italiana) ASI and Italian Ministry of Defence [2]. It is a "dual-use" mission being the overall objective of this program the global Earth observation and the relevant data exploitation for the needs of both the military and the civil (institutional and commercial) communities [3]. The first three satellites have been launched on June, December 2007 and October 2008, respectively. Each of the four satellites is equipped with an SAR operating in X-band with multimode (spotlight, stripmap, and scansar) and multipolarisation capability. 

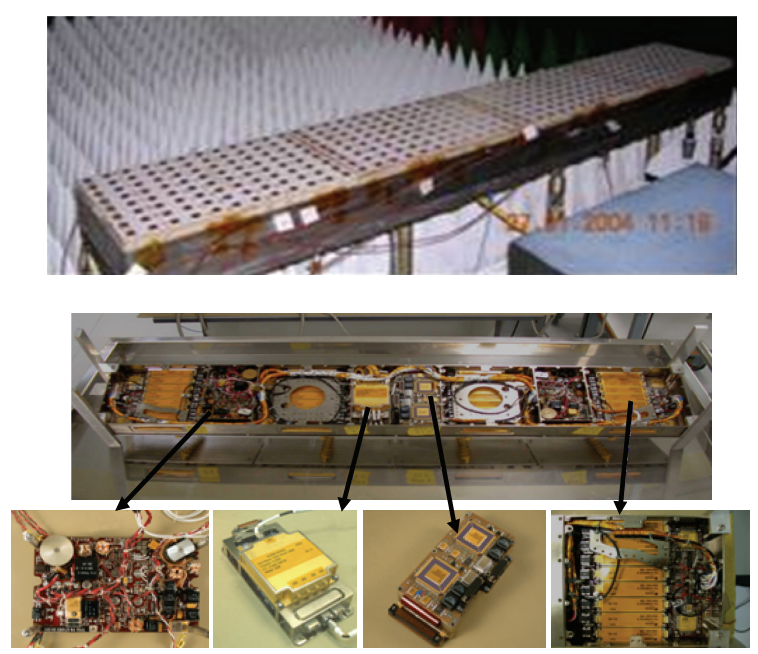

FIgURE 5: Tile external \& internal view: TPSU, TTDL, digital controller and EFE with 8 TR modules are shown (from left to right).

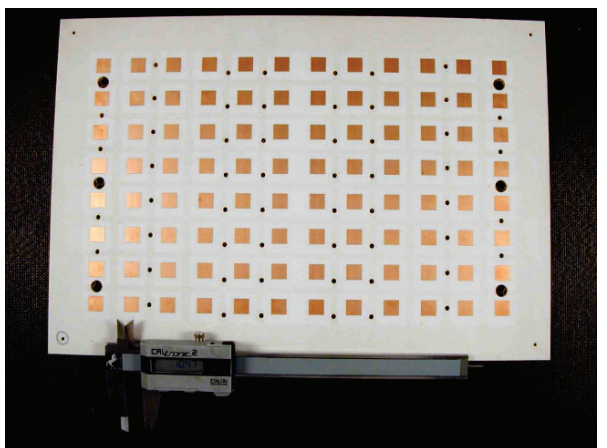

(a) Radiating board front

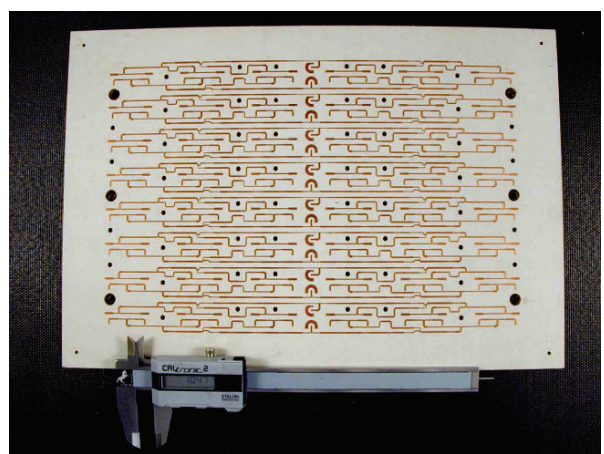

(b) Radiating board back

FIGURE 6: Radiating board: patches side and BFNs side.

The dual-use objective requires for an high operational flexibility (multimode, access area, left and right looking) which is one of the major mission requirements driving the selection of a system based on a phased array, in particular having good steering capabilities on both the elevation and the azimuth planes. In addition to the operational flexibility, the demand on image quality in terms of spatial resolution, swath extension, and ambiguity suppression led to some of the system solutions (instantaneous bandwidth, beam shaping capability in the elevation plane, and beam setting separately in $\mathrm{Tx}$ and $\mathrm{Rx}$ on a pulse repetition frequency basis) which constrained the selected architecture and drove the selection of an active phased array for the antenna subsystem. The protoflight antenna mounted on the satellite in stowed and deployed condition is shown in Figure 2.

The antenna consists of an active phased array organized into five electrical panels, each consisting of a column of 8 Tiles, grouped in three mechanical panels. The electrical panels are grouped into three mechanical panels, two deployable lateral panels with two columns of 8 tiles each, and a fixed central panel, with a single column of tiles, mounted on the S/C top floor. In Figure 3 the antenna organization in panels and tiles is shown.

The tile is the fundamental brick of the antenna [4] and includes linear arrays, TR modules and it is characterized by the presence of the TTDL.

The TTDL is required to stabilize the beam pointing when the beam is steered in azimuth and in elevation. The Tiles are mounted on an aluminum panel frame, which supports also the hold down and release mechanisms (4 for each panel), the deployment mechanisms, the antenna harness, and two beam forming networks (one for the RF $\mathrm{Tx} / \mathrm{Rx}$ distribution and one for calibration purposes).

The digital control of the antenna is achieved by five redounded 1553 digital busses, one for each column of 8 Tiles. The digital distribution of one column of 8 tiles is shown in Figure 4, where the links to the Digital Electronic Sub-System (DESS) main and redundant are indicated. Two 1553 microcouplers are used to allow both the DESS to communicate with the main and redundant Slave Beam Controller sections placed inside each Tile.

Concerning the Tile, two main objectives have been reached during its design and development: 


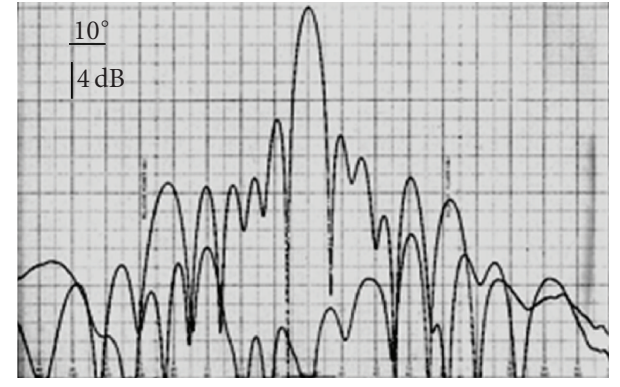

(a)

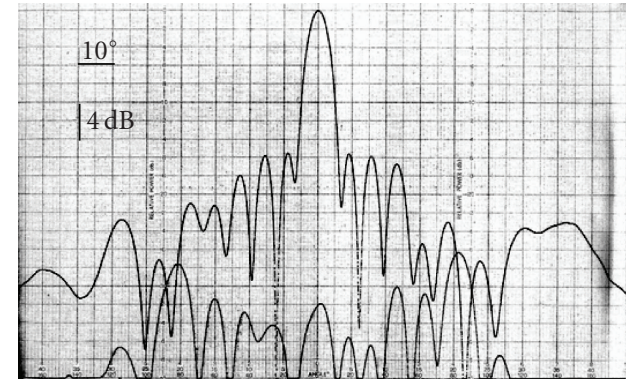

(b)

FIGURE 7: Radiating board measured pattern azimuth plane at $9.6 \mathrm{GHz}, \mathrm{H} \& \mathrm{~V}$ pol. ( $\pm 90^{\circ} x$ axis; $40 \mathrm{~dB} y$ axis).

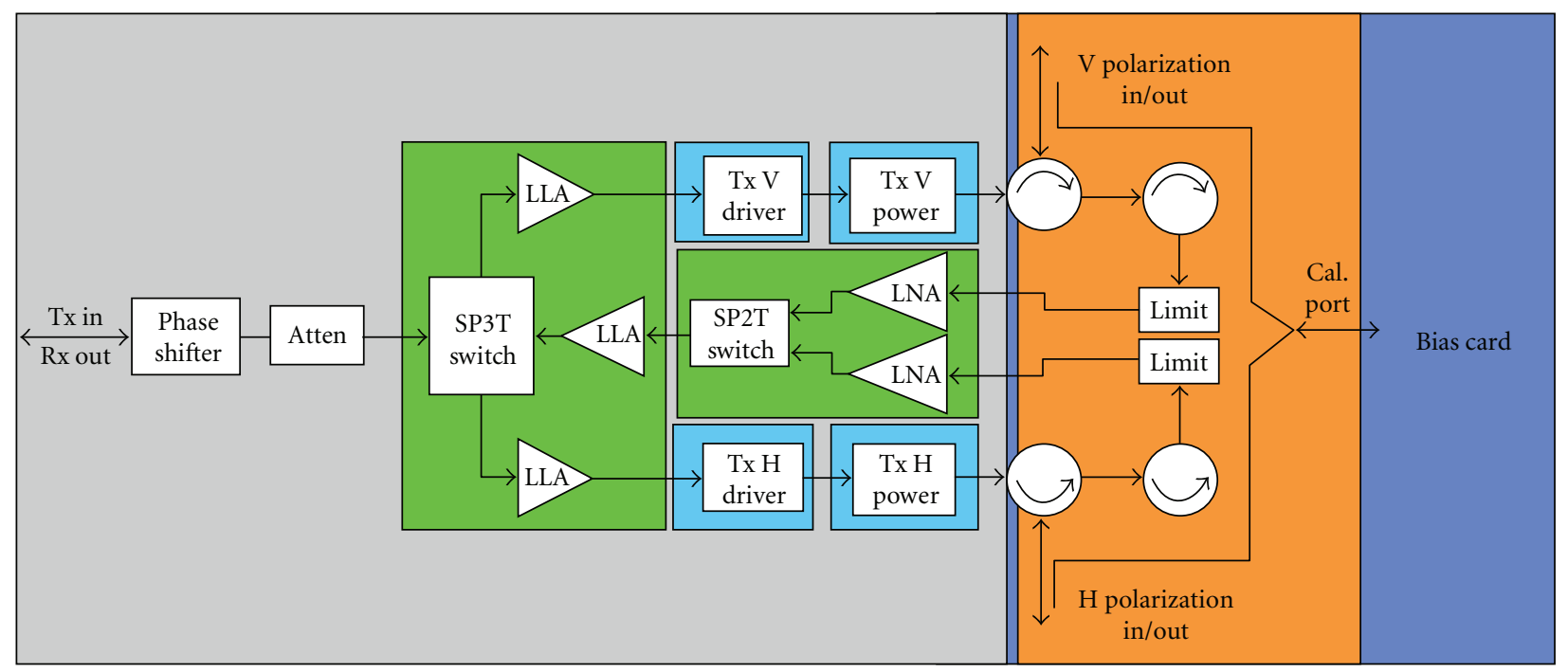

Figure 8: T/R module and RF layout.
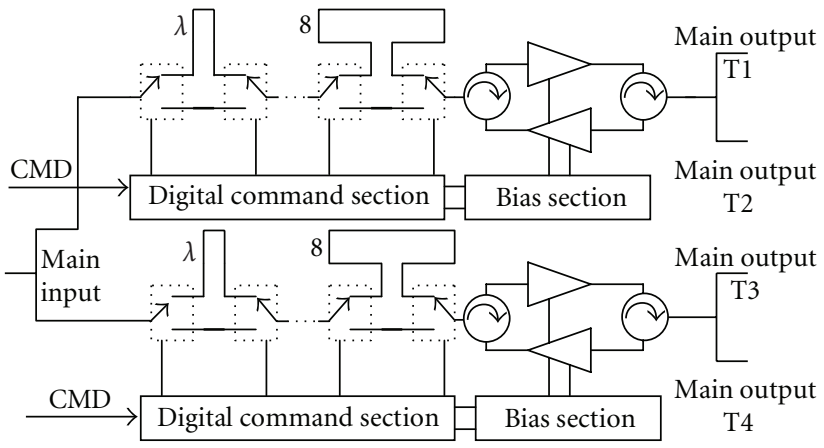

Figure 9: True delay line block diagram.

(i) to design and develop a compact, modular self standing unit;

(ii) to simplify the production line for the antenna.

The first objective has been achieved by including all the electrical and electronic functions inside the unit. This means that the antenna is made of tiles and other passive parts such as harness, BFN, and structure, but no electronics are outside the tile. This allows also to have a modular approach for the antenna: different antennas in size and performance can be achieved just changing the number of tiles, the harness, the beam forming networks and structural parts, but without any modification to the electronics of the tile. The digital controller of each tile compensates in temperature all the RF chains, including TR modules, and RF cables. The TTDL is set according to the beam steering and the tile position in the antenna to stabilize the beam in the frequency band.

Concerning the mechanical design of the tile, a particular effort was spent in the mass reduction of each electrical part, achieving the goal to have a mass less than $10 \mathrm{~kg}$.

The Tile is internally divided into seven separated rooms, four for the T/R modules, two for the power supply units, and one for the digital controller and the TTDL, each closed with a proper cover. The external and internal view of a tile are shown in Figure 5.

The main RF functions of the tile are implemented by the radiating linear arrays, grouped into four radiating boards, able to work in double linear polarisation, by the $\mathrm{T} / \mathrm{R}$ modules, organized in blocks of eight on four EFEs cards, which amplify both the transmitted and received signals and are also able to control amplitude and phase for 


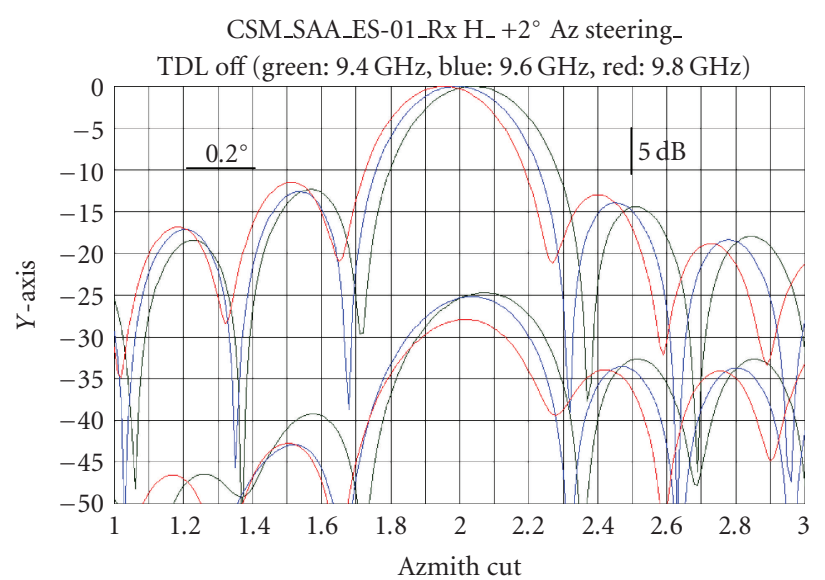

(a)

CSM_SAA_ES-01_Rx H_ $+2^{\circ}$ Az steering-

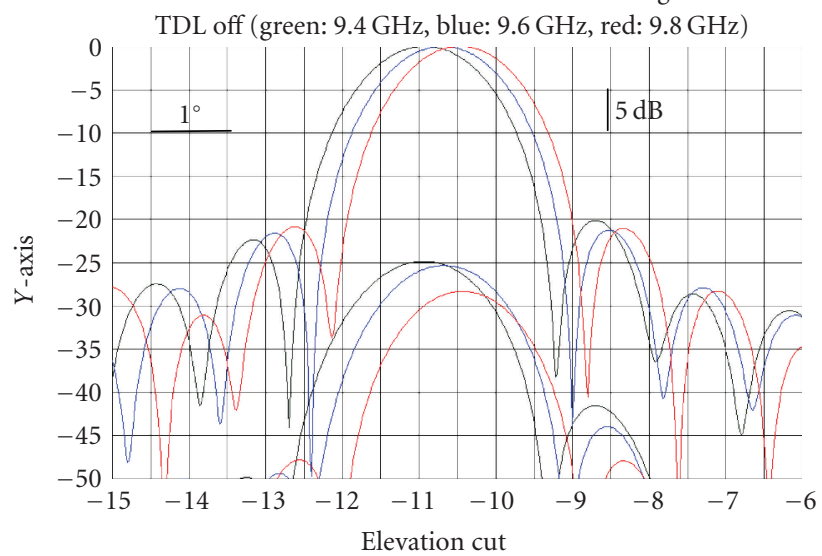

Elevation cut
CSM_SAA_ES-01_Rx H $\mathrm{H}_{-}+2^{\circ}$

Az_RH (red: $9.4 \mathrm{GHz}$, blue: $9.6 \mathrm{GHz}$, green: $9.8 \mathrm{GHz}$ )

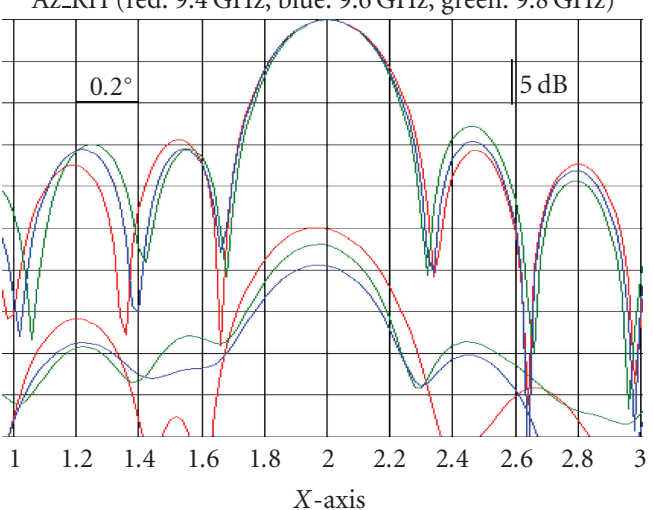

$X$-axis

CSM_SAA_ES-01_RV

(red: $9.4 \mathrm{GHz}$, blue: $9.6 \mathrm{GHz}$, green: $9.8 \mathrm{GHz}$ )

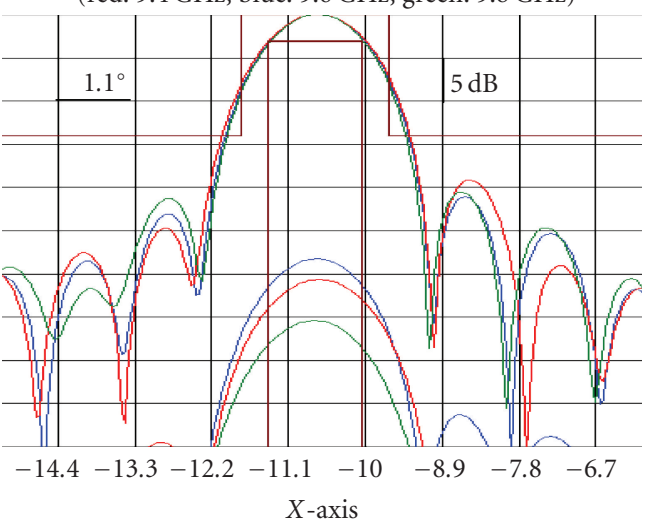

(b)

FIGURE 10: Example of the beam stabilization in the frequency band by using the TTDL (central and extremes frequencies are shown). (a) Azimuth plane ( $+2^{\circ}$ of steering); (b) Elevation plane ( $-10.7^{\circ}$ of steering).

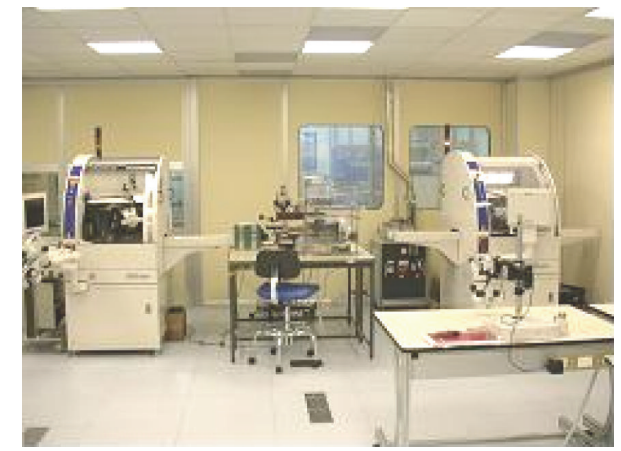

(a)

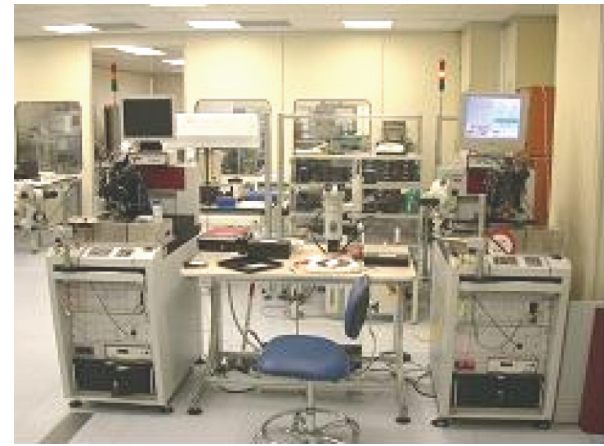

(b)

FIgURE 11: TR module production lines: two in parallel lines were developed to meet the required production rate up to 300 TR modules/month.

beam steering and shaping, and finally by the TTDL which amplifies the RF signals (transmitted and received) and it is able to apply up to 15 wavelength of delay ( 4 bits device) for beam stabilisation in frequency under steering condition.

Concerning the digital functions used to drive the Tile, it is realized by the Slave Beam Controller (SBC). This unit receives commands and beams data from the central digital unit of the Radar by 1553 serial digital bus, and sends to the T/R modules and the TTDL the wanted settings through two parallel serial busses. A look-up table (LUT) is used to compensate their variation in the temperature range. 


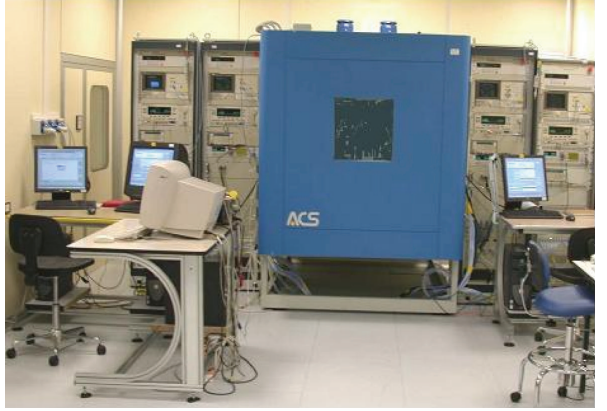

FIgURE 12: Tile production line is shown with 2 ATE (automatic test equipment) working in parallel on two tiles to perform test in temperature of all the $128 \mathrm{RF}$ chains.

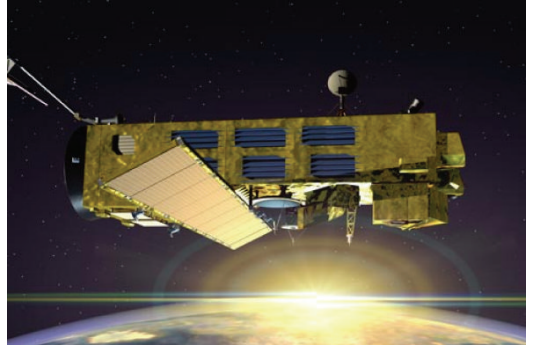

(a)

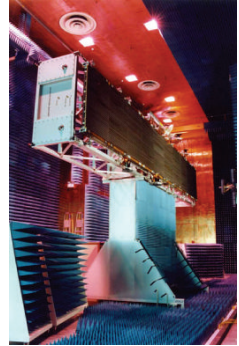

(b)
FIgURe 13: Pictorial view of the ASAR antenna on ENVISAT and in near field.

The power sub-system of the Tile is based on two Tile Power Supply Units, named TPSU, able to feed both the T/R modules, the EFEs cards and the TTDL. The two TPSU's of the Tile are able to supply up to $260 \mathrm{~W}$ to the EFEs, T/R modules and, TTDL, with an efficiency better than $80 \%$ for all the possible thermal conditions and bus voltage.

The mechanical design of the Tile is based on an aluminium box which includes all the electronics and supports the radiating boards. Concerning the thermal design, it has to be pointed out that the antenna functioning will be not continuous but with a limited duration (typically from few to ten minutes per orbit). The thermal design is therefore based on the necessity to control the temperature during this periods considering the dynamic evolution of the temperature. In this case the thermal capacity of the Tile has an important effect on the slope of the temperature increasing. Sunshield is used to improve the thermal isolation towards the sun in right looking orientation (front to sun). The thermal dissipation is achieved though 7 separated covers mounted on the back side of the Tile. (second surface mirrors) SSMs and/or silver Teflon tape are used to improve the thermal dissipation to the deep space. The SSMs improve also the thermal control in the back to sun antenna orientation. The $\mathrm{T} / \mathrm{R}$ modules are mounted by screws to the box, while the thermal exchange with the Tile is improved by thermal filler placed on the hot area of the modules. Hereafter some design and technological aspects of the RF units inside the tile are discussed.
Linear Arrays. The RF radiator consists of a dual-polarized linear array of 12 stacked patches, electromagnetically coupled through slots to the distribution networks. Two distribution networks are used, one for each linear polarization, to feed the 12 stacked patches. Blind mate connectors allow a simple assembly with the active modules. Eight radiating arrays are manufactured together and assembled into a single radiating board. The radiating board is shown in Figure 6, while measured patterns in $\mathrm{H} \& \mathrm{~V}$ polarizations are reported in Figure 7.

The ohmic losses have been minimized by supporting the microstrip dividers with dielectric spacers, avoiding glues over the microstrips. The complete assembly results into a lightweight compact radiating board that can be assembled at tile level by six screws. Bandwidth and mass performance fully meet the given specification of 260 grams per board.

EFE \& T/R Module. The EFE is a printed circuit board (PCB) which is housed in a dedicated room inside the mechanical structure of the Tile. The EFE PCB has two main functions:

(i) digital and power supply distribution,

(ii) RF distribution.

The first is developed on an eight-layer polyimide PCB, supporting the digital and power supply sections and the T/R Modules, while the second is achieved by two layers PCB in PTFE, mechanically fixed (screwed) to the first one.

The T/R module design is based on use of MMICs and includes two Tx channels and two Rx channels to reduce the ohmic losses and to improve the output power and the noise figure. The RF circuit and block diagram of the T/R module is shown in Figure 8.

The T/R module is designed in compliance with requirements needed for space applications.

TTDL. The TTDL is a four-bit device able to reach up to 15 wavelengths of slope compensation. It is based on MMICs switches and microstrip lines. The unit has also the function to amplify both the $\mathrm{Tx}$ and the $\mathrm{Rx}$ signals. The TTDL includes RF dividers to feed the 4 EFEs, each interfacing with $8 \mathrm{~T} / \mathrm{R}$ modules. The True Delay Line block diagram is shown in Figure 9. The Unit provides amplification and phase control on the RF signals between the common I/O port and four I/O ports. In addition, a separate 1:4 divider/combiner is included for the calibration path. The main functions of the unit are

(i) amplify RF signal for recovering the lines losses;

(ii) select the insertion delay between 0 and $15 \lambda$ ( 4 bits);

(iii) receive and decode the digital command data

(iv) bias the amplifiers during the operative part of the period.

The TTDL has to be intended as two separated units which have a common mechanical housing but operating independently, with above mentioned functions and the supply voltages separated. 

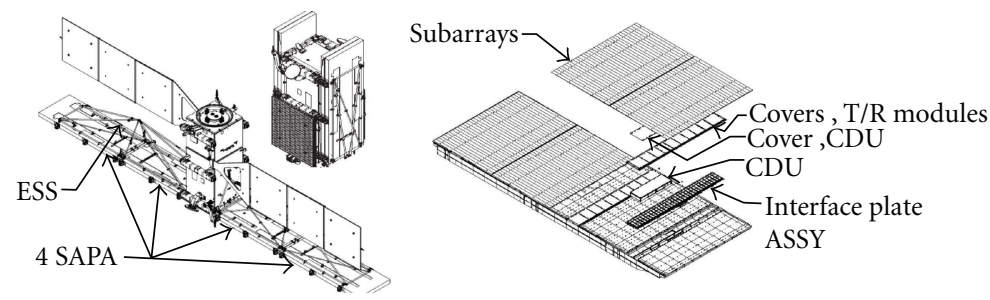

Figure 14: Radarsat 2 antenna, panel and ESS, from [6].

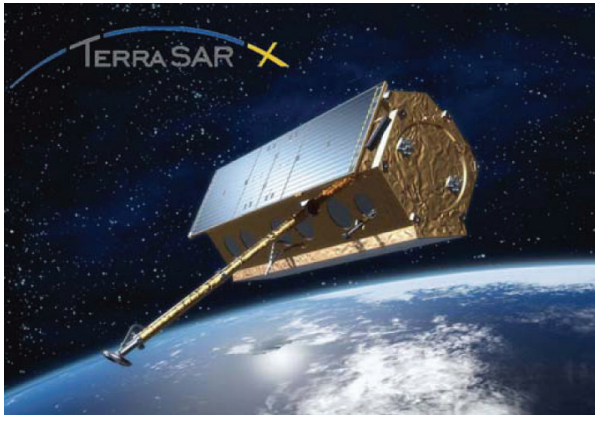

Figure 15: TerrasarX (Astrium Ge) [7].

In Figure 10 the stabilization effects of the TTDL over the antenna beam pointing for both the azimuth and elevation main planes, for $2^{\circ}$ of azimuth steering and $10.7^{\circ}$ of elevation steering are shown ( $4 \%$ of bandwidth).

Production Lines. In order to manufacture and test the 4 antennas for COSMO Sky Med, a dedicated production line for Tiles have been developed. It consists in two main sections:

(i) the TR module production line,

(ii) the Tile production line.

The TR module production is based on two parallel lines each equipped with an automatic pick and place machine and a wire bonding system, as shown in Figure 11.

After to be assembled, the TR module is submitted to an acceptance test campaign which also include an automatic test system.

Concerning the Tiles, also in this case two parallel lines have been developed. The most demanding test is the in temperature characterization/compensation test. Each line is therefore equipped with a thermal chamber and two measurement systems to test in parallel two tiles, as shown in Figure 12.

2.2. Other in Flight SAR Antennas. In the last 10 years other three missions with SAR payload having an active Antenna have been developed and launched for institutional and commercial applications.

(1) ASAR on Envisat Satellite, developed in Europe, under (European Space Agency) ESA contract; the Envisat satellite was launched on 2002;
(2) Radarsat 2, developed by MDA, under (Canadian Space Agency) CSA contract; launched at the end of 2007.

(3) Terrasar X, developed in Germany, funded by the German Aerospace Center (DLR), launched on june 2007;

Apart Envisat, all the other satellites were launched in 2007, demonstrating the increasing of interest and funding by the national space agencies.

All the above SAR Antennas have the typical architecture of the active phased array based on the use of large aperture filled by a number of linear arrays, arranged in columns and rows, each supplied by a Transmit/Receive module. These antennas adopt separate amplitude and phase distribution laws for azimuth plane (typically unifom) and for the elevation plane, where about hundred beams can be generated with different size and steering angles by suitable weighting the amplitude (in Rx mode only) and the phase (in Tx and $\mathrm{Rx}$ ). The three missions use the $\mathrm{C}$ band (ASAR, Radarsat 2) or the $\mathrm{X}$ band (TerrasarX) with bandwidths that ranges from $1 \%$ to $3 \%$. Hereafter a summary of their characteristics is reported as available from the literature [57].

The ASAR Antenna, shown in Figure 13, has an aperture of $10 \mathrm{~m} \times 1.3 \mathrm{~m}$ divided in 10 columns of 32 linear array, [5]. The antenna is organized in five panels with 4 Tiles each, and the $320 \mathrm{TR}$ modules are grouped into 20 Tiles. The panels are in stacked configuration at the launch and deployed by the DEM which includes 4 hinge lines and the relevant step motors. The panels supporting frame is realized in CFRP. It supports the 4 tiles, the RF radiators, the RF distribution network, based on CFRP waveguides, the harness the hold down \& release mechanisms, and the deployment mechanism.

The Tile includes 16 linear arrays mounted on an supporting plate, with the TR modules and the relevant service electronics such as 4 power supplies and the tile controller.

The Radarsat 2 Antenna is $15 \mathrm{~m}$ length and $1.4 \mathrm{~m}$ large [6]. It is organized in two wings of two mechanical panels each, as shown in Figure 14. The panel includes 4 columns of 32 linear arrays. The mechanical panels consists on a aluminum box in which all the RF, power and digital distribution, four columns of TR modules, the relevant service electronics to supply and control the modules, are included. The deployment of the wings is performed by dedicated HRMs and (Extendible Supporting Structure) ESS. 
The Terrasar X satellite, shown in Figure 15, has an Antenna with 384 linear arrays organized in 12 columns of 32 radiators, as described in [7]. Each column of TR modules corresponds to a tile, inside which two power supplies, two digital controllers, harness, and RF distribution are mounted. The dual-polarized linear arrays in waveguide technology realize also the mechanical supporting frame for the tile electronics. The Tiles are directly mounted to a side wall of the satellite.

\section{New Programs}

At the moment new programs are under ways in Europe and in Canada. Concerning the ESA, it is involved in a new program named Sentinel 1. Thales Alenia Space Italy is the Prime contractor and will furnish the satellite and the EFEs with the TR modules in C band for the active antenna which is under responsibility of Astrium Ge, while the Radar electronics is under development in Astrium UK.

In Germany a new mission, named TandemX is under way, which consists in one satellite which will work together with TerrasarX to realize an interferometric mission.

In Italy the manufacturing of the last satellite of Cosmo constellation is on-going, together with the radar payload for (Korean Aerospace Research Institute) KARI, which include an active antenna in $\mathrm{X}$ band.

In Canada the Radarsat Constellation Mission is a followon project to Radarsat 2, currently developed by MDA. The constellation will consist of a fleet of three spacecrafts. The primary purpose of the RCM is to provide C-Band data continuity for RADARSAT-2 users, as well as adding a new series of applications enabled through the constellation approach, such as interferometric mission, improving also the revisit time.

\section{Conclusions}

The paper has summarized the design guidelines followed in Europe and Canada for the development of large active phased array for SAR application. Cosmo Sky Med design and implementation has been also presented. All these antennas have similar radiators arrangement on rectangular grid, except for Cosmo Sky Med which is a dual-use mission and therefore implement a different radiator arrangement to improve also the azimuth steering performance. From mechanical point of view different approaches are mainly due to the extensive use of carbon fiber composite material for linear arrays and supporting structure from one side, in opposition to aluminium-based configurations developed on the same frequencies and for similar antenna dimensions. While the advantages for thermal aspects are clear for the aluminium case, the CFRP-based solution can guarantee a major aperture stability, at the expense of lower thermal conductivity. Comparison among these antennas have shown also a similar approach based on tile arrangement, the larger difference is for Cosmo Sky Med which utilizes also TTDL for beam stabilization in steering and tiles staggering to improve the grating lobes control.
The main development ongoing are directed to improve the revisit time of the satellite on observed area of interest, moving towards to the constellation systems as minimum to perform radar interferometry and to improve the revisit time for commercial images application.

\section{Acknowledgments}

The Author wishes to thank the Italian Space Agency (ASI) and the Minister Of Dsefence for the support given by during the whole development of COSMO Sky Med program, and the whole Thales Alenia Space Team placed in Rome, L'Aquila, Milan, and Turin for the large efforts demonstrated during the design, development and production of COSMO SAR Antennas.

\section{References}

[1] R. C. Hansen, Phased Array Antennas, John Wiley \& Sons, New York, NY, USA, 1998.

[2] F. Caltagirone, G. Angino, A. Coletta, F. Impagnatiello, and A. Gallon, "COSMO-SkyMed program: status and perspectives," in Proceedings of the 3rd International Workshop on Satellite Constellations and Formation Flying, Pisa, Italy, February 2003.

[3] F. Caltagirone, P. Spera, G. Manoni, and L. Bianchi, "COSMOSkyMed: a dual use earth observation constellation," in Proceedings of the 2nd International Workshop on Satellite Constellations and Formation Flying, Haifa, Israel, February 2001.

[4] P. Capece, L. Borgarelli, M. Di Lazzaro, U. Di Marcantonio, and A. Torre, "COSMO SkyMed active phased array SAR instrument," in Proceedings of IEEE International Radar Conference (RADAR '08), pp. 1-4, Rome, Italy, May 2008.

[5] R. Torres, "ASAR instrument stability," in Proceedings of the CEOS Working Group on Calibration/Validation-SAR Workshop, pp. 42-48, London, UK, September 2002.

[6] S. Riendeau and C. Grenier, "RADARSAT-2 antenna," in Proceedings of the IEEE Aerospace Conference, pp. 1-9, Big Sky, Mont, USA, March 2007.

[7] S. Buckreuss, R. Werninghaus, and W. Pitz, "The German satellite mission TerraSAR-X," in Proceedings of IEEE International Radar Conference (RADAR '08), pp. 1-5, Rome, Italy, May 2008. 

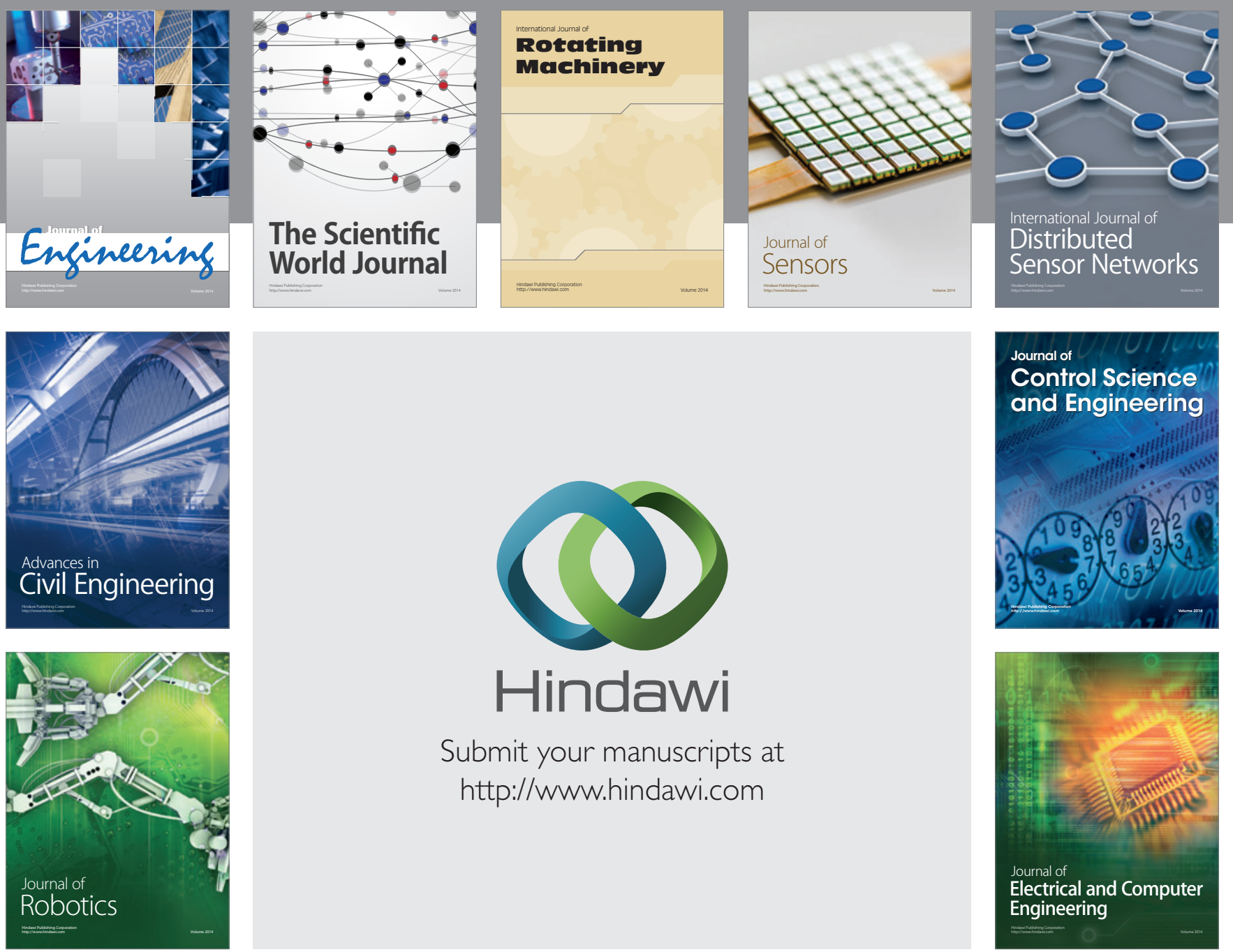

Submit your manuscripts at

http://www.hindawi.com
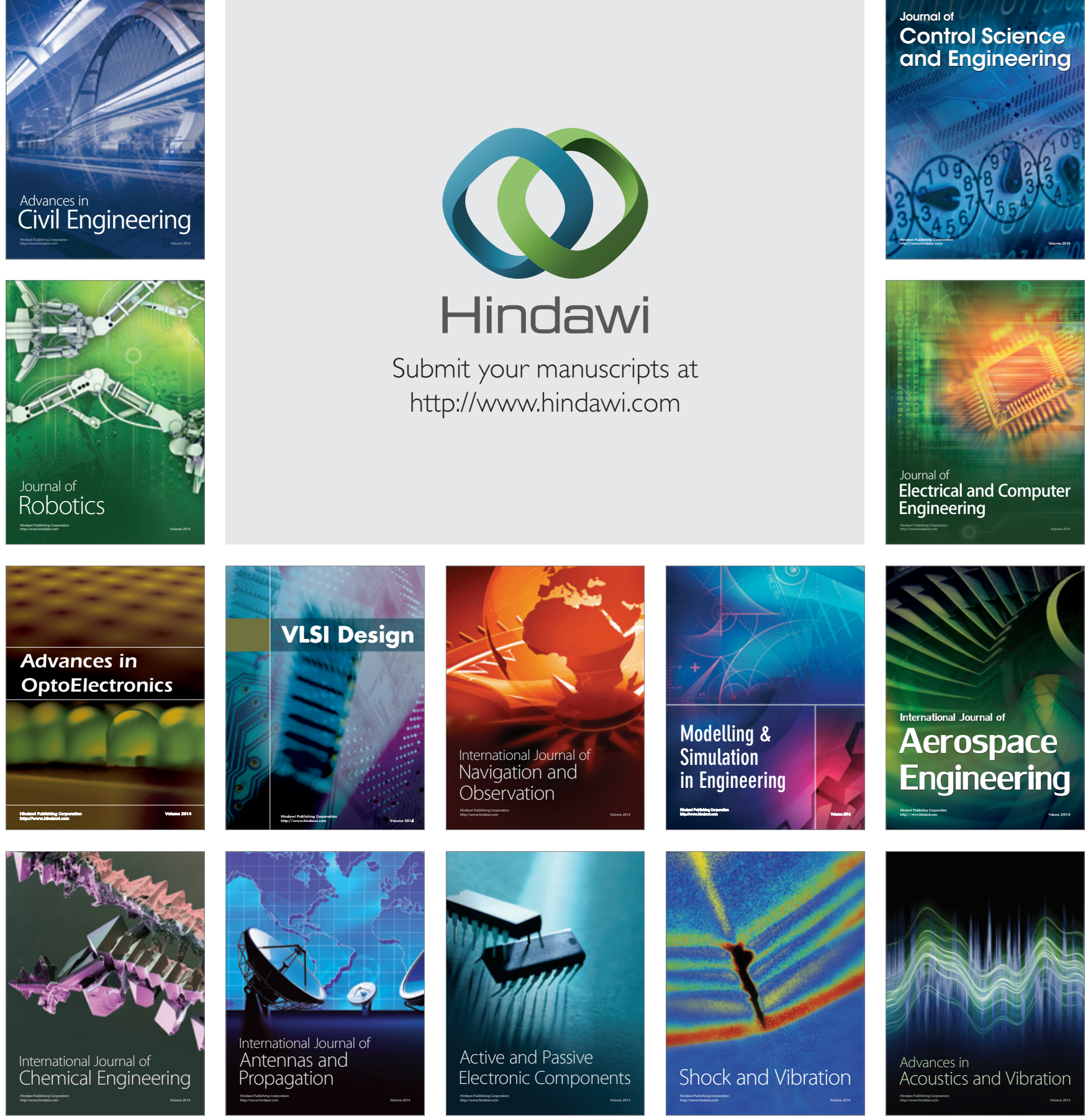